\title{
Discordância da Lipoproteína de Baixa Densidade e da Lipoproteína de Alta Densidade com Gravidade de Doença Arterial Coronariana
}

\author{
Discordance of Low-Density Lipoprotein Cholesterol and Non-High-Density Lipoprotein Cholesterol with \\ Severity of Coronary Artery Disease
}

\author{
Iran Castro ${ }^{1}$ e Hugo Fontana Filho ${ }^{1}$
}

Instituto de Cardiologia - Fundação Universitária de Cardiologia, ${ }^{1}$ Porto Alegre, RS - Brasil

Minieditorial referente ao artigo: Discordância entre Colesterol LDL e Não-HDL e Gravidade da Doença Arterial Coronária

As doenças cardiovasculares (DCVs) são as principais causas de morte em todo o mundo. ${ }^{1}$ A dislipidemia é um fator de risco e também um fator causal de DCVs, sendo foco da terapia de prevenção primária e secundária das DCVs.

Existe um consenso e um amplo conhecimento acerca dos mecanismos causais da lipoproteína de baixa densidade (LDL) nas DCVs, e os benefícios da terapia hipolipemiante, sendo que a magnitude de seu efeito é proporcional à redução dos níveis circulantes. ${ }^{2}$ No entanto, apesar do uso intensivo de agentes hipolipemiantes, permanece um risco residual, um alvo constante de pesquisa e terapia.

Evidência recente confirma que o evento inicial da aterogênese é a retenção de LDL e outras partículas na parede do vaso. ${ }^{3}$ Níveis elevados de colesterol não-HDL ajudam a identificar pacientes que continuam com alto risco para eventos cardiovasculares apesar de níveis baixos de LDL. ${ }^{4}$

\section{Palavras-chave}

Doenças Cardiovasculares/mortalidade; Lipoproteinas LDL; Lipoproteinas HDL; Doença da Artéria Coronariana; Inibidores de Hidroximetilglutaril-CoA Redutases; ProProteina Convertase 9.

Correspondência: Iran Castro •

Instituto de Cardiologia - Métodos Gráficos - Av. Princesa Isabel, 395.

CEP 90620-000, Santana, Porto Alegre, RS - Brasil

E-mail: irancstro@gmail.com

DOI: https://doi.org/10.36660/abc.20200033

\section{Referências}

1. Organização Mundial da Saúde; Organização Pan-Americana da Saúde. (OPAS/OMS) [Internet]. Doenças cardiovasculares. (acesso em 2020 jan 11). Disponível em: <https://www.paho.org/bra/index.php?option=com content\&view $=$ article \&id $=5638: 10$-principais-causas-de-morte-nomundo\&Itemid $=0$

2. Silverman MG, Ference BA, Im K, Wiviott SD, Giugliano RP, Grundy SM, et al. Association Between Lowering LDL-C and Cardiovascular Risk Reduction Among Different Therapeutic Interventions: A Systematic Review and Metaanalysis. JAMA. 2016;316(12):1289.

3. Ference BA, Ginsberg HN, Graham I, Ray KK, Packard CI, Bruckert E, et al . Lowdensity lipoproteins cause atherosclerotic cardiovascular disease. 1. Evidence from genetic, epidemiologic, and clinical studies. A consensus statement from the European Atherosclerosis Society Consensus Panel. Eur Heart J. 2017;38(32):2459-72.

4. Arsenault BJ, Boekholdt SM, Kastelein JJ. Lipid parameters for measuring risk of cardiovascular disease. Nat Rev Cardiol. 2011;8(4):197-206.
O presente estudo ${ }^{5}$ avaliou retrospectivamente características anatômicas de 574 pacientes diagnosticados com síndrome coronariana aguda e correlacionou os achados com níveis séricos de LDL e não-HDL. Observou-se uma discordância de $15 \%$ entre LDL e não-HDL, o que foi similar a estudos prévios. ${ }^{6}$ No entanto, não foram identificadas diferenças anatômicas significativas na avaliação da gravidade da doença aterosclerótica. O uso prévio de estatinas tem um efeito mais significativo na redução de LDL em comparação a não-HDL, o que pode explicar a falta de associação.

Além disso, a sensibilidade dos níveis de LDL em identificar risco cardiovascular é menor no diabetes. ${ }^{7}$ De fato, o presente estudo identificou discrepâncias na associação entre diabetes mellitus e níveis de LDL. Talvez o tamanho da amostra não tenha sido adequado para identificar diferenças anatômicas entre os grupos. O acompanhamento de pacientes com associações discrepantes poderia identificar um subgrupo de pacientes em alto risco para novos eventos cardiovasculares.

As diretrizes brasileiras já incluem metas de LDL e não-HDL, buscando identificar indivíduos com níveis adequados de LDL e alto risco residual para eventos cardiovasculares. Estudos prospectivos certamente ajudarão a identificar o subgrupo de pacientes que requeiram uma abordagem terapêutica mais intensa, e que provavelmente se beneficiariam de terapias mais caras e efetivas, como inibidores de PCSK9 e lomitapida. ${ }^{8}$ Estudos anteriores já mostraram a eficácia desses medicamentos na redução de eventos cardiovasculares e apolipoprotein(a) em alguns grupos de pacientes.

5. Kurmus O, Erkan AF, Ekici B, Aslan T, Eren M. Discordance of Low-Density Lipoprotein Cholestrol and Non-High-Density Lipoprotein Cholestrol and Coronary Artery Disease Severity. Arq Bras Cardiol. 2020; 114(3):469-475

6. Sniderman AD, Islam S, Yusuf S, McQueen MJ. Discordance analysis of apolipoprotein B and non-high density lipoprotein cholesterol as markers of cardiovascular risk in the INTERHEART study. Atherosclerosis. 2012;225(2):444-9.

7. Mark L, Vallejo-Vaz AJ, Reiber I, Paragh G, Kondapally Seshasai SR, et al Non-HDL cholesterol goal attainment and its relationship with triglyceride concentrations among diabetic subjects with cardiovascular disease: a nationwide survey of 2674 individuals in Hungary. Atherosclerosis. $2015 ; 241(1): 62-8$.

8. Kim SH, Baek SH. Lomitapide, relief pitcher for patients with homozygous familial hypercholesterolemia. Eur J Prev Cardiol.2019; 27(2) 155-6. 